\title{
SCIDoC
}

\author{
International Journal of Dentistry and Oral Science (IJDOS) \\ ISSN: 2377-8075
}

\section{Evaluation For Survival Rate of Implants in Medically Compromised Individuals}

Research Article

Shilpa Shetty ${ }^{1}$, Swarup Shetty ${ }^{2 *}$, Naveen Reddy $\mathrm{R}^{3}$, Dhruv Arora ${ }^{4}$, Pareedhi Arora ${ }^{5}$, Kaushik Shetty $B^{6}$

${ }^{1}$ Assistant Professor, Department of Prosthodontics and Crown \& Bridge, Sharavathi Dental College and Hospital, Alkola, Shimoga, Karnataka, India.

${ }^{2}$ Associate Professor, Department of Prosthodontics and Crown \& Bridge, Sharavathi Dental College and Hospital, Alkola, Shimoga, Karnataka, India.

${ }^{3}$ Assistant Professor, Department of Prosthodontics, College of Dentistry, Jazan University, Saudi Arabia.

${ }^{4}$ Prosthodontics and Implantology, Sen Lecturer, Department of Prosthodontics, Seema Dental College and Hospital, HNBG University, Rishikesh, Uttarakhand, India.

${ }^{5}$ Oral \& Maxillofacial Surgery, Consultant Oral \& Maxillofacial Surgeon, New Delhi, India.

${ }^{6}$ Lecturer, Department of Orthodontics and Dentofacial Orthopedics, Nitte (Deemed to be University), AB Shetty Memorial Institute of Dental Sciences (ABSIMDS), Mangalore, Karnataka, India.

\section{Abstract}

Introduction: The success of implant procedure is depending on operator's skill, proper selection of case and underlying medical conditions.

Materials and Methods: This retrospective study was done on 60 subjects with 30 from each group; Group A with medical conditions and Group B with healthy individuals as control group. Amount of bone loss around the implant over $1 \mathrm{~mm}$ of bone loss after one year and over $0.3 \mathrm{~mm}$ bone loss at each succeeding year were measured as failures. The implant failure was confirmed based on clinical and radiographic evaluation at follow up visits.

Results: Total 48 implants were placed in 30 medically compromised patients, whereas 46 implants placed in 30 control group. Implant failure was 8, 3, 2 and 1 in diabetes, cardiovascular disease, hypothyroidism and ectodermal dysplasia cases respectively. Diabetes patients had higher implant failure compared to other medical conditions. Total $14(29.2 \%)$ implants were failed in group A, and only $2(0.043 \%)$ implants were failed in control group after 5 years of follow up. Bone loss was $1.32 \mathrm{~mm}$ in group A and $0.4 \mathrm{~mm}$ in group B after 1 year $(<0.01)$ and $3.1 \mathrm{~mm}$ in group A and $1.2 \mathrm{~mm}$ in group B after 5 years $(<0.001)$. Conclusion: From the present study it was concluded that, diabetic patients had higher failure rate compared to other medical conditions. Implants survival was greater in healthy subjects compared to those with medical conditions.

Keywords: Cardiovascular; Diabetes; Implant; Medical Conditions; Survival Rate.

\section{Introduction}

Dental caries and periodontal diseases are the common reason for tooth loss. Dental implant rehabilitation is the best treatment options for replacement of missing teeth.[1] Over past 10 years' dental implant had higher success rate of $90 \%$ to $95 \%$. The reasons for implants failure are; lack of osseointegration during early healing, infection of the peri-implant tissues, and due to underlying medical conditions. The placement of dental implant is quite simple and easy in healthy individual as associated to unhealthy subjects. In medically compromised patients such as patients with hypertension, diabetes, hypothyroidism, severe bleeding disorders etc., special care has to be taken before placing implant. $[1,2]$

A medically compromised patient (MCP) can be described, "as the one who has a distinctive physical or mental feature regarding the people of the same age. medical history allows us to identify the systemic disease and the success rate expected in the MCP that is going to be rehabilitated with dental implants". Improvements in surgical technique has increased survival rate of dental implants in some types of medically compromised conditions. [3] Type of bone, amount of bone, length of edentulous jaw segment, hidden pathologies such as root pieces, inflammatory processes etc., play vital role in implant success. Systemic conditions

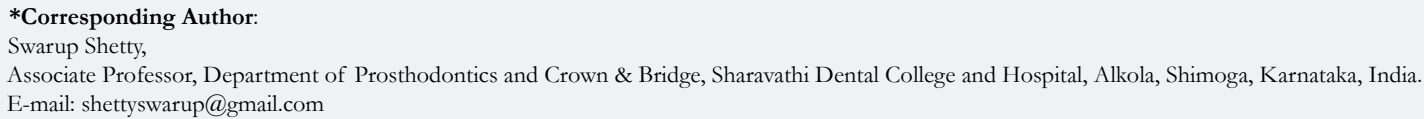

Citation: Shilpa Shetty, Swarup Shetty, Naveen Reddy R, Dhruv Arora, Pareedhi Arora, Kaushik Shetty B. Evaluation For Survival Rate of Implants in Medically Compromised Individuals. Int J Dentistry Oral Sci. 2021;8(8):3674-3677. doi: http://dx.doi.org/10.19070/2377-8075-21000752

Copyright: Swarup Shetty 2021 . This is an open-access article distributed under the terms of the Creative Commons Attribution License, which permits unrestricted use, distribution and reproduction in any medium, provided the original author and source are credited. 
such as hypothyroidism, diabetes, mellitus, bleeding disorders, thyrotoxicosis, xerostomia, smoking, osteoporosis, ectodermal dysplasia CVS etc., are few conditions which pose challenge to dental implant treatment. Absolute contraindications for implant placement is consist of myocardial infarction and cerebrovascular accident, cardiac transplant, immunosuppression, active treatment of malignancy, drug abuse, and psychiatric disorders. $[1,2,4,5]$

There are very few reported studies on survival of implant in medically compromised individuals. Hence the present study was carried out to measure implant failure rate in patients with various medical conditions in comparison to healthy patients.

\section{Material and Method}

This study was conducted in the department of Prosthodontics and Oral implantology after attaining clearance from institutional ethics committee. Informed consent was obtained from all the participants. This retrospective study was done on 60 subjects with 30 from each group; Group A with medical conditions and Group B with healthy individuals as control group.

Inclusion criteria comprised of patient's age ranged 35-65 years, patients with comprehensive medical and dental history and patients who received dental implant 5 years ago. Exclusion criteria consisted of patients with history of chemotherapy, radiation therapy, cancer therapy and with improper records.
Demographic data such as name, gender, age, etc., were collected from the patient's data file. Over $1 \mathrm{~mm}$ of bone loss around the implant after one year and over $0.3 \mathrm{~mm}$ bone loss at every succeeding year were considered as failures. The confirmation of implant failure was made based on clinical and radiographic evaluation at follow up visits. All the relevant clinical and radiographic information of each patient was attained from the recorded files. The study was done by single trained investigator.

The obtained data was statistically evaluated with SPSS package (21.0 version, Inc.; Chicago, IL) using Mann-Whitneytest, chi square test at $\mathrm{P}$ value less than 0.05 was considered significant.

\section{Results}

Table 1 indicated that, age group 35-45 comprised of 10 patients in group A and 7 in group B, 46-55 years had 12 in group A and 10 in group $B$ and 56-65 years had 8 in group $A$ and 13 in group B. Total 48 implants were placed in 30 medically compromised patients whereas 46 implants placed in 30 control healthy subjects. Table 2 indicates distribution of implants and implant failures with various medical conditions. In diabetic patients (10) had 18 implants, Cardiovascular disease patients (8) had 10 implants, hypothyroidism (6) had 11 implants, ectodermal dysplasia (4) had 6 and Osteoporosis (2) had 3 implants $(\mathrm{P}<0.05)$.Implant failure was $8,3,2$ and 1 in diabetes, cardiovascular disease, hypothyroid-

Table 1. Patient distribution.

\begin{tabular}{|c|c|c|c|}
\hline Age range in years & Group A & Group B & $\mathbf{p}$ \\
\hline $35-45$ & 10 & 7 & 0.13 \\
\hline $46-55$ & 12 & 10 & 0.12 \\
\hline $56-65$ & 8 & 13 & 0.01 \\
\hline Total & 30 & 30 & \\
\hline
\end{tabular}

Mann-Whitney test, $\mathrm{P}<0.05$ was significant

Table 2. Distribution of implants and failures with various medical conditions.

\begin{tabular}{|c|c|c|c|c|}
\hline Various medical conditions & Number patients & Number implants & Implants failure & $\mathbf{p}$ \\
\hline Diabetes & 10 & 18 & 8 & 0.052 \\
\hline Cardio vascular diseases & 8 & 10 & 3 \\
\hline Hypothyroidism & 6 & 11 & 2 \\
\hline Ectodermal dysplasia & 4 & 6 & 1 \\
\hline Osteoporosis & 2 & 3 & 0 \\
\hline Total implants & 30 & 48 & 14 & \\
\hline
\end{tabular}

Test used: Chi- square test, $\mathrm{P}<0.05$ was significant.

Table 3. Failure rate of implants in both the groups.

\begin{tabular}{|c|c|c|c|}
\hline Implant failure & Group A & Group B & $\mathbf{p}$ \\
\hline Number & $14(29.2 \%)$ & $2(0.043 \%)$ & 0.001 \\
\hline Mean bone loss in mm after 1 year & 1.32 & 0.4 & 0.01 \\
\hline Mean bone loss in mm after 5 years & 3.1 & 1.2 & 0.001 \\
\hline
\end{tabular}

Chi- square test, $\mathrm{P}<0.05$ was significant 
ism and ectodermal dysplasiacases respectively. Diabetes patients had higher implant failure compared to other medical conditions. Table 3 indicates, failure rate of implants in both the groups. Total $14(29.2 \%)$ implants were failed in group A, and only $2(0.043 \%)$ implants were failed in control group after 5 years of follow up, and it was statistically highly significant. Bone loss was $1.32 \mathrm{~mm}$ in group $A$ and $0.4 \mathrm{~mm}$ in group B after 1 year $(<0.01)$ and $3.1 \mathrm{~mm}$ in group $A$ and $1.2 \mathrm{~mm}$ in group B after 5 years $(<0.001)$.

\section{Discussion}

Dental implant in medically compromised patients is challenge for dental surgeon. General health plays an important role in confirming the success of dental implant treatment. Conditions like diabetes mellitus, hypertension, osteomyelitis, oral cancer, and mental disability pose challenges as the general health of patients is compromised.[1]

Controlled or non-controlled diabetic patients are more prone for periodontitis due to infection, compromised host defense systems, microvascular disease which adversely affect the blood supply. [6]

Patients with prosthetic valvular replacement are usually susceptible for infective endocarditis. Due to the high risk of complications following a myocardial infarction or cerebrovascular accident, the dental practitioners should wait until preliminary stabilization. Patient may pursue elective dental care only after 6 months of ischemic incident with medical clearance. Cardiac patient under anticoagulant or thrombolytic therapy may interfere with implant procedures.[7]

Infection, pain and hemorrhage and occasionally neuropathy are initial complications of implant. Implants have got failure rates also. Failure is typically because of loosening, breakage, or infection but complications can include pain or occasionally neuropathy. [8]

Ectodermal dysplasia includes a heterogeneous group of genetic disorders with incidence 100,000 live births. It disturbs ectodermal structures and it is related to hypo/anodontia. ectodermal dysplasia associated with severe and early onset periodontitis. [9]

Osteoporosis is a very common skeletal disease characterized by a reduction in bone density and alterations in the microstructure of bone that lead to an increased risk of fractures. Thus osteoporosis could impair implant success. Osteoporosis, metabolic disease which modifies the bone mass and density. [3] However, with use of modified, hydrophilic surfaced implant treatment can improve the success of dental implant.[9] Bisphosphonates inhibit bone resorption, and, thus, treat osteoporosis.7But use of antiresorptive drugs, such as bisphosphonates and denosumab, is accompanied by an increased risk of developing so-called medicationrelated osteonecrosis of the jaws. [4]

It has been suggested that diabetes mellitus and other medical conditions reduce the immunity of patients. The healing capacity of body decreases especially in diabetic patients. Hence special consideration should be given to these patients.[10] Cancer patients taking ionizing radiation and chemotherapy can disrupt host defense mechanisms and hematopoiesis. Because the pa- tient on such regimens cannot shows an appropriate response to wound healing from surgery. Addictions to alcohol and other drugs, however, lower resistance to disease, increase possibility of infection. [7]

Present study was done to assess the survival rate of implants in medically compromised individuals over healthy one after 5 years of follow up. In present study diabetes patients had higher implant failure compared to other medical conditions. Total 14 $(29.2 \%)$ implants were failed in group A, and only $2(0.043 \%)$ implants were failed in control group after 5 years of follow up. Bone loss was $1.32 \mathrm{~mm}$ in group A and $0.4 \mathrm{~mm}$ in group B after 1 year $(<0.01)$ and $3.1 \mathrm{~mm}$ in group $\mathrm{A}$ and $1.2 \mathrm{~mm}$ in group B after 5 years $(<0.001)$.

Parihar et al evaluated the failure rate of dental implant in patients with various medical conditions in comparison of healthy patients after implant placement. They concluded that diabetic patients had higher failure rate in comparison to other medical conditions. [1] Gómez-de Diego et al review the current scientific literature in order to analyze the indications and contraindications of dental implants in medically compromised patients. They suggested that, Cardiac systemic diseases, diabetic endocrine pathologies or controlled metabolic disorders do not seem to be a total or partial contraindication to the placement of dental implants. [3]

Khajuria et al evaluated the outcomes of dental implants in medically compromised patients. The failure rate of dental implants among the patients was $2 \%$ in group I and $7 \%$ in group II. The difference was statistical significant $(\mathrm{P}<0.05) .2$ The results are similar to our findings. Jagadeesh et al evaluated the hort dental implants survival rate of in medically compromised subjects in comparison to healthy one using short implants. They concluded that those with medical conditions had more implant failure compared to healthy one.[10] Dutt et al evaluated the dental implants survival rates in medically compromised participants. Similar to our findings they found lower success rate of implant sin medically compromised individuals over healthy one.8Syed Ismail et al assessed the failure rate of dental implant in medically compromised individuals compared to healthy one. Similar to our findings they found higher failure rate of implant in diabetic patients.[5]

Millesi et al with retrospective follow- up evaluated the long- term survival of implant patients with diabetes, bisphosphonate therapy and osteoporosis. They didn't find significant implant failures in all the three group during follow up visit, which is in contrast to our findings.[11]

Diz et al assessed the survival rates of dental implants in medically compromised patient. They concluded that individualized medical control should be established prior to implant therapy, since in many of these patients the quality of life and functional benefits from dental implants may outweigh any risks.[12] Manor et al investigate the rate of complications and failures following dental implantation in medically compromised patients. They found a similar rate of failure and complications of dental implantation in medically complex patients and in healthy patients. [13] In contrast to our study Alsaadi and col analyzed seven systemic diseases in a retrospective study and concluded that diabetic endocrine pathology is not associated with a higher frequency of failure in dental implants.[14] 
In the present study lower implant success was observed with various medical conditions and diabetic patients had higher failure rate compared to other medical conditions. Implants survival was greater in healthy subjects compared to those with medical conditions.

The drawback of the present study is smaller samples size for shorter duration and different types of medical conditions were not included. Further long term studies are needed to validate the implant survival.

\section{Conclusion}

From the present study it was concluded that, various medical conditions can affect the survival of dental implants and diabetic patients had higher failure rate compared to other medical conditions. Implants survival was greater in healthy subjects compared to those with medical conditions.

\section{References}

[1]. Parihar AS, Madhuri S, Devanna R, Sharma G, Singh R, Shetty K. Assessment of failure rate of dental implants in medically compromised patients. J Family Med Prim Care. 2020 Feb;9(2):883-5.

[2]. Khajuria R, Sudan T, Sudan S, Sharma S. Assessment of dental implants in medically compromised patients: A retrospective study. Int. j. community health med. res. 2018;4(1):54-56.

[3]. Gómez-de Diego R, Mang-de la Rosa Mdel R, Romero-Pérez MJ, CutandoSoriano A, López-Valverde-Centeno A. Indications and contraindications of dental implants in medically compromised patients: update. Med Oral Patol Oral Cir Bucal. 2014 Sep 1;19(5):e483-9.Pubmed PMID: 24608222.

[4]. Vissink A, Spijkervet FK, Raghoebar GM. The medically compromised patient: Are dental implants a feasible option?. Oral Dis. 2018 Mar;24(12):253-60.

[5]. Syed Ismail PM, Ravi S, Mohammed Saif T, Madhumala R, Sayee Ganesh N, Manovijay B, et al. Evaluation of Dental Implants Failure Rate of in Patients with Various Medical Conditions. Int J Cur Res Rev. 2021;13(5):S81-S-83.

[6]. Beikler T, Flemmig TF. Implants in the medically compromised patient. Crit Rev Oral Biol Med. 2003 Jul;14(4):305-16.

[7]. Hwang D, Wang HL. Medical contraindications to implant therapy: part I: absolute contraindications. Implant Dent. 2006 Dec 1;15(4):353-60.

[8]. Dutt P, Srivastava V, Chand P, Singh BP, Jurel SK. A retrospective study of assessment of survival rates of dental implants in medically compromised patients. J App Dent Med Sci. 2018;4(1):171-175.

[9]. Donos N, Calciolari E. Dental implants in patients affected by systemic diseases. Br. Dent. J. 2014 Oct;217(8):425-30.

[10]. Jagadeesh KN, Verma AK, Parihar AS, Kochhar AS, Das AC, Razi MA. Assessment of the Survival Rate of Short Dental Implants in Medically Compromised Patients. J Contemp Dent Pract. 2020 Aug 1;21(8):880-883.Pubmed PMID: 33568609.

[11]. Millesi W, Baboun R, Fürhauser R, Mailath-Pokorny G, Busenlechner D, Haas R, et al. 10-year survival analysis of dental implants in medically compromised patients-diabetes, osteoporosis and bisphosphonates. Clin Oral Impl Res. 2018 Oct; ;29(Suppl. 17):49.

[12]. Diz P, Scully C, Sanz M. Dental implants in the medically compromised patient. . J Dent. 2013 Mar 1;41(3):195-206.

[13]. Manor Y, Simon R, Haim D, Garfunkel A, Moses O. Dental implants in medically complex patients-a retrospective study. Clin. Oral Investig. 2017 Mar 1;21(2):701-8.

[14]. Alsaadi G, Quirynen M, Komárek A, van Steenberghe D. Impact of local and systemic factors on the incidence of late oral implant loss. Clin Oral Implants Res. 2008 Jul;19(7):670-6.Pubmed PMID: 18492080. 